\title{
Relation entre la vitesse relative de croissance et la teneur en azote chez la laitue (Lactuca sativa $\mathbf{L}$ ) Effets du rayonnement et de la température
}

\author{
L Dapoigny $^{1}$, A Fleury ${ }^{2 *}$, P Robin ${ }^{3}$ \\ 'Chaire des sciences et techniques des productions légumières, ENSH, 2, rue Le Nôtre, F-49045 Angers; \\ ${ }^{2}$ Laboratoire de recherche : agriculture urbaine, ENSP, 4, rue Hardy, RP 914, F-78009 Versailles ; \\ ${ }^{3}$ Unité de recherche en écophysiologie et horticulture, Inra, domaine Saint-Paul, Agroparc, F-84914 Avignon cedex 9, France
}

(Reçu le 21 août 1996 ; accepté le 4 décembre 1996)

\begin{abstract}
Summary - Relation between relative growth rate and nitrogen content during growth in lettuce (Lactuca sativa $\mathbf{L}$ ). Effects of radiation and temperature. The relation between relative growth rate of fresh matter and reduced nitrogen content in this fresh matter was studied during the growth of the lettuce in summer and in autumn. Four treatments for each season: two nutrional levels and two radiation levels have been applied to plants fed with nutrient solution and grown in sand in a plastic greenhouse. The level of radiation has no effect on the relation between the relative growth rate and the nitrogen content. For the same nitrogen content the relative growth rate in summer expressed in day ${ }^{-1}$ was twice that in autumn. They became the same if the relative growth rate, which correlates positively with temperature, was calculated over time expressed in degree.day. These results show the importance of nitrogen content and temperature on the determination of the relative growth rate.
\end{abstract}

reduced nitrogen / relative growth rate / lettuce / temperature

Résumé - La relation entre la vitesse relative de croissance de la matière fraîche et la teneur en azote réduit dans cette même matière fraîche a été étudiée entre le semis et la maturité commerciale chez la laitue cultivée sous abri pour deux cultures : l'une en été et l'autre en automne. Pour chaque saison, quatre traitements (deux niveaux nutritionnels croisés avec deux niveaux lumineux) ont été appliqués à des plantes ferti-irriguées en conditions de hors-sol sur sable. Le niveau de rayonnement ne montre pas d'effet sur la relation entre la VRC et la teneur en azote réduit. À même teneur en azote, les vitesses relatives de croissance d'été sont doubles de celles de l'automne. Les relations saisonnières deviennent semblables si la vitesse relative de croissance, qui présente une relation linéaire positive avec la température, est calculée en fonction de la durée exprimée en degré.jour. Ces résultats soulignent l'importance conjointe de la température et de la teneur en azote réduit dans l'établissement d'une vitesse relative de croissance.

azote réduit / vitesse relative de croissance / Lactuca sativa $\mathrm{L}$ = laitue / température

* Correspondance et tirés à part.

Tél : (33) 0139246273 ; fax : (33) 0139246274

Abréviations : MF : matière fraîche ; PAR : rayonnement photosynthétiquement actif ; es : erreur standard de l'estimation, VRC :

vitesse relative de croissance 


\section{INTRODUCTION}

Une relation linéaire entre la vitesse relative de croissance (VRC), exprimée en fonction de la matière fraîche, et la teneur en azote réduit dans la matière fraîche a été mise en évidence au cours de la croissance de la laitue (Dapoigny et al, 1996). Cette relation a été déterminée pour une culture d'été, quand la plante présente une croissance rapide. Cependant, pour être utile comme outil de modélisation, la relation entre la VRC et la teneur en azote réduit doit être validée dans un contexte climatique plus large. En effet, pour une teneur en azote donnée, le climat est susceptible d'induire, selon le rayonnement et la température, des vitesses relatives de croissance différentes. En particulier, le rayonnement reçu par la plante, auquel l'accumulation de matière sèche est proportionnelle (Gosse et al, 1986), peut modifier la relation entre la vitesse relative de croissance et la teneur en azote. Cependant les résultats précédents (Dapoigny et al, 1996) n'ont pas montré d'effet de l'ombrage sur cette relation. Qu'en serait-t-il pour une gamme de rayonnement plus faible limitant la croissance? Par ailleurs, la température semble avoir un effet important : pour un même rayonnement, mais à deux régimes distincts de température, Caloin et Yu $(1984,1986)$ observent des relations très différentes entre la VRC et la teneur en azote. Plusieurs travaux montrent les multiples incidences de la température sur les processus de croissance (Wheeler et al, 1993a, 1993b; Lawlor et al, 1988). Son importance sur les processus de développement et en conséquence sur les processus de croissance a conduit certains auteurs à utiliser une mesure thermochronique du temps (Lemaire et Salette, 1981; Fleury et al, 1986, Scaife et al, 1987).

L'objectif de cet article est de tenter une généralisation de la relation observée entre la VRC et la teneur en azote réduit de la plante pour différentes conditions climatiques de culture. Des laitues ont été cultivées en été et en automne avec deux niveaux d'alimentation minérale croisés avec deux niveaux de rayonnement. La croissance de la laitue est étudiée en fonction de sa teneur en azote réduit au cours des deux saisons différenciées par les valeurs de rayonnement et de température

\section{MATÉRIELS ET MÉTHODES}

\section{Cultures et traitements}

Les cultures hors sol de laitue sous tunnel sont conduites en pots sur sable, avec deux niveaux de solu- tion nutritive ( $\mathrm{N} 1$ et $\mathrm{N} 2$ ), croisés avec deux niveaux de rayonnement $(\mathrm{R} 1=60 \% \mathrm{R} 2)$ selon des conditions qui ont été décrites précédemment (Dapoigny et al, 1996). Les quatre traitements (RIN1, RIN2, R2N1, R2N2) sont appliqués du repiquage à la récolte avec deux répétitions.

Deux cultures sont réalisées : une en été (Cycle 1 , semis le $21 / 07 / 94$, repiquage le $02 / 08 / 94$, récolte le 06/9/94), une en automne (Cycle 2, semis le 26/09/94, repiquage le $12 / 10 / 94$, récolte le $22 / 12 / 94$ pour le traitement $\mathrm{N} 2$ et le 3/01/95 pour le traitement $\mathrm{N} 1$ ).

\section{Données climatiques}

Pour chaque niveau de rayonnement, la température est mesurée au niveau des plantes à l'aide de deux sondes au platine (PT 100) par traitement, reliées à une centrale d'acquisition. Des mesures de rayonnement photosynthétiquement actif (PAR) sont effectuées au cours de la culture à l'aide de capteurs à l'arséniure de gallium (Pontallier, 1990). Les mesures sont réalisées toutes les six minutes, puis la moyenne journalière est calculée.

Les rayonnements photosynthétiquement actifs moyens journaliers sont compris : pour l'été en éclairement naturel R2, entre 434 et $170 \mu \mathrm{mol} . \mathrm{s}^{-1} \cdot \mathrm{m}^{-2}$ avec une moyenne de $297 \mu \mathrm{mol} . \mathrm{s}^{-1} \cdot \mathrm{m}^{-2}$, et sous ombrage $\mathrm{R} 1$, entre 254 et $109 \mu \mathrm{mol} . \mathrm{s}^{-1} \cdot \mathrm{m}^{-2}$ avec une moyenne de $181 \mu \mathrm{mol} . \mathrm{s}^{-1} \cdot \mathrm{m}^{-2}$; pour l'automne en R2 entre 282 et $16 \mu \mathrm{mol} . \mathrm{s}^{-1} \cdot \mathrm{m}^{-2}$ avec une moyenne de $97 \mu \mathrm{mol}$. $\mathrm{s}^{-1}, \mathrm{~m}^{-2}$. et sous ombrage $\mathrm{Rl}$ entre 201 et $12 \mu \mathrm{mol}$. $\mathrm{s}^{-1} \cdot \mathrm{m}^{-2}$ avec une moyenne de $64 \mu \mathrm{mol} . \mathrm{s}^{-1} \cdot \mathrm{m}^{-2}$. Les températures moyennes journalières varient en été de 19 à $30^{\circ} \mathrm{C}$ avec une moyenne de $24,1^{\circ} \mathrm{C}$ pour l'ensemble de la culture, en automne de 4 à $21^{\circ} \mathrm{C}$ avec une moyenne de $12,4^{\circ} \mathrm{C}$ pour l'ensemble de la culture. Entre les saisons, les rayonnements moyens sur les cycles varient du simple au quadruple, les températures moyennes du simple au double. Pour une saison donnée, l'ombrage de $40 \%$ (R1) n'ayant pas modifié le régime thermique, la corrélation prévisible entre le rayonnement et la température, est réduite de fait.

\section{Mesures de biomasse et de la vitesse relative de croissance}

Douze prélèvements sont réalisés entre le repiquage et la maturité commerciale à des intervalles variant entre 2 et 10 jours suivant le développement de la culture. À chaque prélèvement et pour chaque traitement, deux lots de quatre plantes sont récoltés au lever du soleil. Les parties aériennes et les racines sont pesées individuellement avant et après séchage $\left(48\right.$ heures $\left.80^{\circ} \mathrm{C}\right)$. La vitesse relative moyenne de croissance est calculée pour chaque période comprise entre deux prélèvements en appliquant la formule $\left(\operatorname{Ln} \mathrm{P}_{2}-\operatorname{Ln} \mathrm{P}_{1}\right) /\left(\mathrm{t}_{2}-\mathrm{t}_{1}\right), \mathrm{P}_{2}$ et $\mathrm{P}_{1}$ étant les masses de matière fraîche aérienne respectivement aux temps $t_{2}$ et $t_{1}$ (Venus et Causton, 
1979). La durée entre deux prélèvements est évaluée en jour ou degré. jour.

\section{Dosage d'azote}

Les analyses des teneurs en azote sont réalisées sur la matière sèche aérienne totale de quatre plantes réunies en un même lot. L'azote total est mesuré après combustion par un analyseur automatique (Leco SP 428, MI, États-Unis) selon la méthode Dumas. Le nitrate est extrait à l'eau et dosé par chromatographie ionique (Dionex Dx 300, États-Unis) avec détection conductimétrique. L'azote réduit est calculé par différence entre l'azote total et l'azote nitrique, l'azote ammoniacal étant considéré comme négligeable.

\section{Analyse statistique}

L'hypothèse classique du modèle linéaire entre la VRC et la teneur en azote est proposée afin de tester l'effet de l'ombrage et de la saison. Les programmes MGLH et Non Linear Model de Systat v5 (Systat for Windows : Statistics, Version 5 Edition Systat, Inc, 1992, Evanston, IL, États- Unis) sont utilisés pour les calculs statistiques. Le niveau classique de probabilité à 0,05 pour le test des différentes hypothèses est utilisé.

\section{RÉSULTATS ET DISCUSSION}

La saison affecte fortement la croissance de la laitue : pour le traitement R2N2, il faut 30 jours de croissance en été pour atteindre la maturité commerciale et l'obtention d'une laitue de $350 \mathrm{~g}$ de matière fraîche, alors que 70 jours sont nécessaires en automne (fig 1). Le rayonnement et la température ont induit des vitesses différentes de croissance, ce qui permet d'étudier, pour des conditions climatiques variées, la relation entre la vitesse relative de croissance et la teneur en azote réduit dans la matière frâ̂che. L'effet de l'alimentation minérale, déjà abordé dans un article précédent (Dapoigny et al, 1996), ne sera pas discuté.

\section{Relation entre la vitesse relative de croissance $\left(\mathbf{j}^{-1}\right)$ de la biomasse fraîche et la teneur en azote réduit dans la matière fraîche}

À même teneur en azote réduit dans la matière fraîche, les VRC de l'été sont doubles de celles de l'automne (fig 2). Cette différence est importante durant la phase juvénile de la plante caractérisée par des teneurs élevées en azote réduit, supérieures à $2 \mathrm{mg}$ Nréd. g $\mathrm{MF}^{-1}$. Avec l'avancement

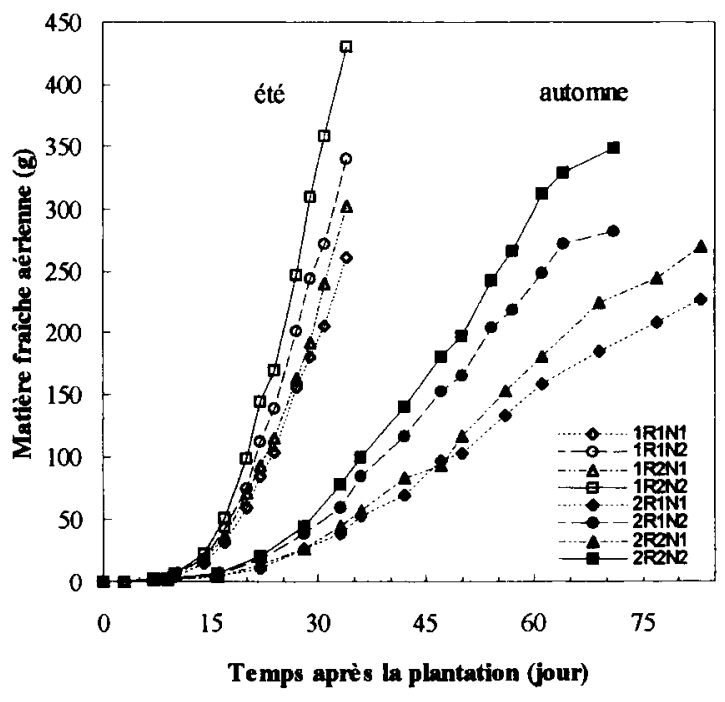

Fig 1. Évolution de la matière fraîche aérienne après plantation pour une culture de laitue en été (symbole ouvert) et en automne (symbole fermé) conduites en pot sur sable aveo solution nutritive avec une alimentation minérale élevée (N2) ou faible (N1) en éclairement naturel (R2) ou réduit de $40 \%$ (R1).

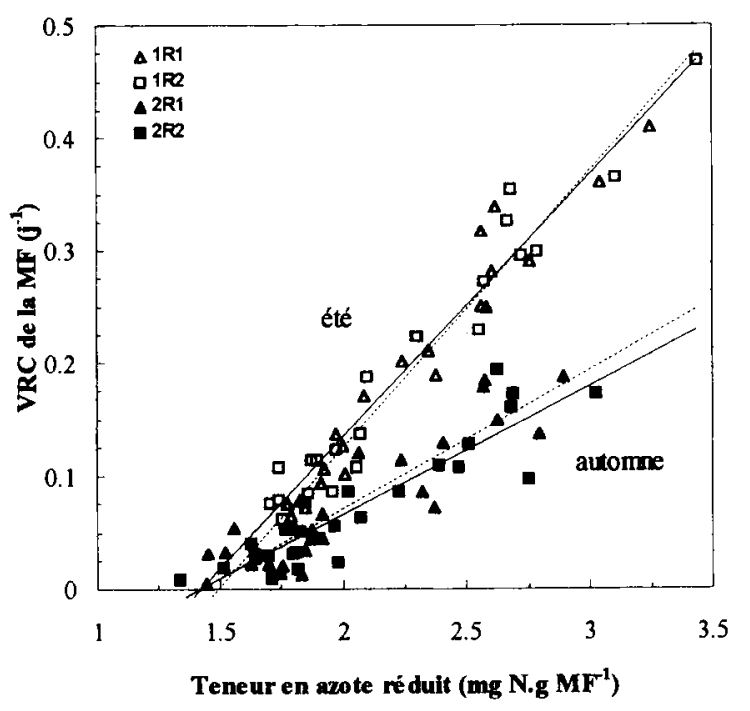

Fig 2. Relation entre la vitesse relative de croissance de la matière fraîche en jour ${ }^{-1}$ et la teneur en azote réduit dans la matière fraîche chez la laitue cultivée sous tunnel en été (symbole ouvert) ou en automne (symbole fermé), sur pot avec un éclairement naturel (carré) ou avec un éclairement naturel réduit de $40 \%$ (triangle). Les pentes et les coefficients de détermination des droites de régression pour l'été et l'automne en éclairement naturel (-) et en éclairement réduit de $40 \%$ ( - - ) sont respectivement en été pour Rl de 0,246, (es $=0,012, \mathrm{ddl}=20, r^{2}=0,95$ ) et pour R2 de 0,233 (es $\left.=0.012, \mathrm{ddl}=20, r^{2}=0,95\right)$, en automne pour $\mathrm{R} 1,0,123$ (es $\left.=0,011, \mathrm{ddl}=24, r^{2}=0,83\right), p<0,001$.

de la culture et la diminution de la teneur en azote dans la plante (Lemaire et Salette, 1984), les VRC de deux saisons diminuent et convergent. 


\section{Effet du rayonnement}

Pour la culture d'été comme pour la culture d'automne, les plantes poussant sous l'éclairement naturel R2 et celles poussant sous l'ombrage R1, s'inscrivent dans une même relation linéaire : en été, la pente est de 0,246 pour $\mathrm{R} 1$ et de 0,233 pour $\mathrm{R} 2$, et est double de celle de l'automne avec respectivement 0,123 pour $\mathrm{R} 1$ et 0,113 pour $\mathrm{R} 2$; les pentes pour les deux niveaux de rayonnement d'une même saison ne sont pas significativement différentes.

Cet absence d'effet du niveau de rayonnement (R1 et R2) sur la relation entre la VRC de la matière fraîche et la teneur en azote réduit est observée pour une large gamme de rayonnement, puisque les valeurs moyennes sur l'ensemble de la culture sont respectivement pour les traitements $\mathrm{R} 1$ et $\mathrm{R} 2$ en automne, de $64 \mu \mathrm{mol} . \mathrm{s}^{-1} \cdot \mathrm{m}^{-2}$ et $97 \mu \mathrm{mol}$. $\mathrm{s}^{-1} \cdot \mathrm{m}^{-2}$, et pour les traitements $\mathrm{R} 1$ et $\mathrm{R} 2$ en été, respectivement de $181 \mu \mathrm{mol} . \mathrm{s}^{-1}$. $\mathrm{m}^{-2}$ et $297 \mu \mathrm{mol}$. $\mathrm{s}^{-1} \cdot \mathrm{m}^{-2}$. La présence d'un faible niveau de rayonnement en automne exclut la possibilité d'une absence d'effet causée par le dépassement du rayonnement optimum pour la photosynthèse. L'ombrage entraîne une baisse de croissance indiquant bien que le rayonnement est limitant. Cette absence d'effet du rayonnement au cours de la saison sur cette relation peut s'expliquer par une modification conjointe de la vitesse relative de croissance et de la teneur en azote réduit. En effet, l'augmentation du rayonnement renforce la croissance, et l'augmentation de la biomasse étant associée à une diminution de la teneur en azote dans cette biomasse (Lemaire et Salette, 1984 ; Greenwood et al, 1986, 1991), la vitesse relative de croissance diminue en conséquence (Greenwood et al, 1990). Ainsi, la relation dynamique entre la VRC et la teneur en azote reste la même quel que soit le niveau de rayonnement (Ingestad et McDonald, 1989), mais les plantes sous fort rayonnement « parcourent » plus rapidement cette relation.

\section{Effet de la température}

En plus de la baisse du niveau de rayonnement, l'automne présente une température plus faible qu'en été. La température moyenne sur l'ensemble de la culture d'été est de $24,1^{\circ} \mathrm{C}$, celle de l'automne de $12,4^{\circ} \mathrm{C}$. Il faut donc tester l'hypothèse que le régime thermique puisse être à l'origine des différentes relations saisonnières entre la VRC de la matière fraîche et la teneur en azote réduit. L'ensemble des deux cultures s'est déroulé d'août à janvier, ce qui entraîne une corrélation positive contingente, du fait de leur très large variation, entre le rayonnement et la température $\left(r^{2}=0,77\right)$. La baisse du rayonnement par l'ombrage réduit la corrélation pour une saison donnée, mais ne l'élimine pas sur les deux cultures prises ensemble. Pour déterminer si la température a un effet prépondérant sur la VRC par rapport au rayonnement, une analyse des coefficients de corrélation partielle est nécessaire. Ainsi, l'analyse de la régression multiple de la VRC $\left(j^{-1}\right)$ en fonction de la teneur en azote réduit, de la température moyenne et/ou du rayonnement moyen, a été réalisée. Le coefficient de corrélation partielle de la VRC en fonction du rayonnement, compte tenu de la teneur en azote réduit et de la température est de 0,038 $(\mathrm{P}=0,713)$, tandis que celui de la VRC en fonction de la température, compte tenu de la teneur en azote réduit et du rayonnement, est de 0,494 ( $p$ $<0,001)$. Ainsi, cette analyse vérifie bien l'absence d' " effet rayonnement $»$ et montre l'influence prépondérante de la température sur la relation entre la VRC et la teneur en azote réduit. Cela confirme les résultats de Caloin et Yu (1984, 1986) qui observent pour une teneur en azote donnée, un effet positif de la température sur la VRC $\left(j^{-1}\right)$.

L'effet de la température moyenne aérienne sur la VRC peut être étudié en comparant les deux saisons. Cependant, l'augmentation de biomasse étant associée à une diminution de la VRC (Greenwood et al, 1986), il faut tenir compte de la biomasse de la plante. En procédant à une analyse en fonction de «phases » de croissance distinguées arbitrairement par des classes de biomasse fraîche, l'effet de la température sur la VRC peut être observé (fïg 3).

Les pentes des droites de régression pour les classes de biomasse fraîche $\mathrm{P}_{\mathrm{MF}}<50 \mathrm{~g}, 50<\mathrm{P}_{\mathrm{MF}}$ $<150 \mathrm{~g}, 150<\mathrm{P}_{\mathrm{MF}}<250 \mathrm{~g}$, et $\mathrm{P}_{\mathrm{MF}}>250 \mathrm{~g}$ sont respectivement de $0,017\left(r^{2}=0,84\right), 0,009$ $\left(r^{2}=0,77\right), 0,005\left(r^{2}=0,84\right)$ et de $0,005\left(r^{2}=0,95\right)$ avec $p<0,001$. La diminution de la pente au cours de la croissance est directement l'expression de la diminution de la teneur en azote réduit. Une relation de proportionnalité apparaît alors entre la VRC et la température, quelle que soit l'alimentation minérale, N1 ou N2. Cet effet positif de la température sur la VRC rejoint d'autres observations faites sur la laitue (Scaife, 1973; Wheeler et al, 1993a) et sur le maïs (Fleury et al, 1986) et confirme l'effet prépondérant de la température sur la croissance (Lawlor et al, 1988). La relation linéaire proposée entre la VRC et la température est une simplification du modèle de Scaife (1973) qui adopte une forme sigmoïdale. Cependant cet 

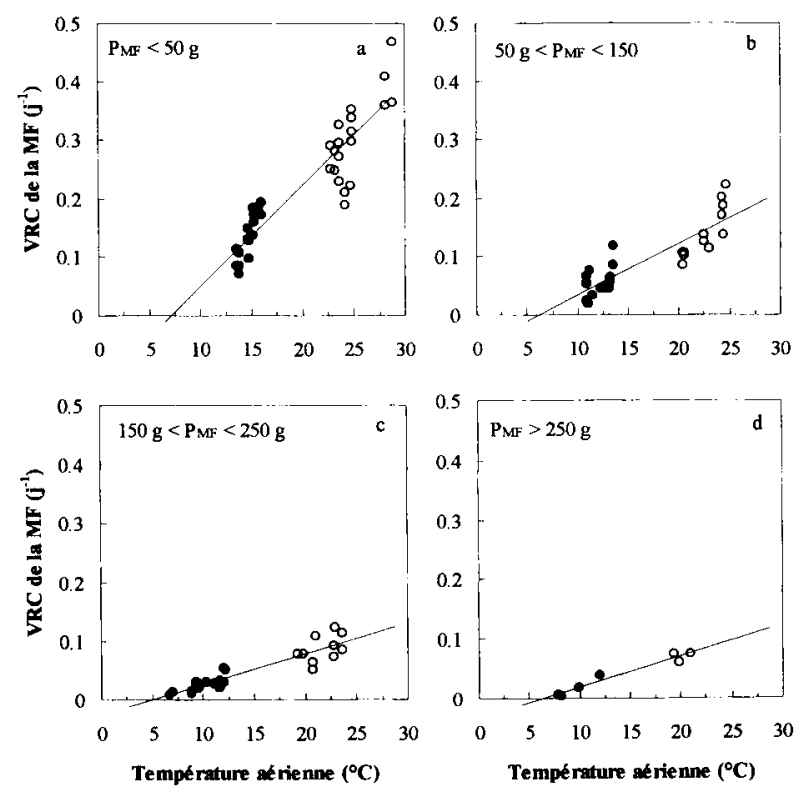

Fig 3. Relation entre la vitesse relative de croissance en matière fraîche exprimée en (jour) ${ }^{-1}$, et la température aérienne moyenne ambiante de la période considérée, chez la laitue cultivée sous tunnel en été (symbole ouvert) ou en automne (symbole fermé), sur pot, et ayant une masse de matière fraîche compris entre 0 et $50 \mathrm{~g}$ (a), 50 et $150 \mathrm{~g}$ (b), 150 et $250 \mathrm{~g}$ (c), ou supérieur à $250 \mathrm{~g}(\mathbf{d})$; les coefficients de détermination $r^{2}$ des droites de régression sont respectivement de 0,$84 ; 0,77 ; 0,84 ; 0,95(p<0,001)$.

auteur se restreint à de jeunes plantes en phase exponentielle de croissance, alors que nos résultats concernent l'ensemble de la phase végétative du cycle de la laitue et présentent une diminution de la pente de la relation au cours de la croissance.

L'interception des droites de régression avec l'abscisse suggèrent une température seuil pour la croissance située entre 5 et $7^{\circ} \mathrm{C}$. En toute rigueur, il serait préférable d'obtenir une évaluation de la température seuil dans des conditions de croissance où seule la température serait modifiée ; cette figure ne peut donc servir à définir la température minimale de croissance pour laquelle les valeurs citées dans la littérature sont de $0^{\circ} \mathrm{C}$ (Wurr et Fellows ; 1984), et de $3,5^{\circ} \mathrm{C}$ (Scaife et al, 1987). Pour déterminer cette température seuil, il faut comparer la VRC de plantes ayant une même potentialité de croissance dans des conditions où la température serait le facteur principal de croissance. La prise en compte de la teneur en azote réduit de la plante autorisant une telle comparaison, il devient alors possible de déterminer la température seuil de la laitue indépendamment de la saison. La formalisation de la VRC en fonction de la teneur en azote réduit de la plante et de la température est alors la suivante :

$$
\operatorname{VRC}\left(\mathrm{j}^{-1}\right)=\mathrm{a} *(\mathrm{~T}-\mathrm{b}) *(\mathrm{Nréd}-\mathrm{c})
$$

avec : T, la température moyenne de la période considérée $\left({ }^{\circ} \mathrm{C}\right)$; Nréd, la teneur en azote réduit dans la matière fraîche (mg Nréd. $\left.\mathrm{g} \mathrm{MF}^{-1}\right)$; $\mathrm{a}$, b et $c$ sont des constantes, où b correspond à la température seuil pour la croissance, et c, à la teneur en azote réduit minimale de croissance.

L'estimation des paramètres fournit l'équation : $\operatorname{VRC}\left(\mathrm{J}^{-1}\right)=0,009 *(\mathrm{~T}-3,013) *(\mathrm{Nréd}-1,229)$, avec $r^{2}=0,94, \mathrm{ddl}=95$.

La température seuil de $3^{\circ} \mathrm{C}$ (es $=0,978$ ) est bien en accord avec les valeurs précitées dans la littérature.

\section{Relation entre la vitesse relative de croissance (degré. jour $^{-1}$ et la teneur en azote réduit}

Ainsi, compte tenu de l'effet de la température sur la VRC exprimée en (jour) ${ }^{-1}$, il apparaît légitime d'utiliser la VRC en (degré. jour) ${ }^{-1}$ comme l'avaient déjà réalisé Scaife et al (1987) dans le cas de jeunes plants de laitue. Dans notre travail, cette analyse est étendue sur l'ensemble de la culture de la laitue et appliquée à la relation entre la VRC et la teneur en azote réduit (fig 4). La température seuil de $3^{\circ} \mathrm{C}$ qui vient d'être déterminée, est utilisée pour définir le temps thermique. Les pentes des droites de régression obtenues durant l'été et l'automne, respectivement de 9,407 et de 8,908 , ne sont alors pas significativement différentes ; la pente de la droite de régression commune pour l'ensemble de deux cultures est de 9,238 (se $\left.=0,382, \mathrm{ddl}=94, r^{2}=0,86, p<0,001\right)$. Des plantes à même teneur en azote réduit présentent, quelle que soit la saison, une même VRC exprimée en (degré. jour) ${ }^{-1}$ (fig 4).

Cette uniformisation des relations est à rapprocher de l'effet de la température sur le développement des feuilles. L'augmentation de la température accélère la production du nombre de feuilles (Bensink, 1971), la vitesse d'élongation des feuilles (Bensink, 1971 ; Lorenz et Wiebe, 1980) et par conséquence la surface foliaire qui détermine le rayonnement photosynthétiquement actif intercepté. Les basses températures vont alors ralentir le développement de la surface d'interception du rayonnement et diminuer la VRC.

\section{CONCLUSION}

Il est possible de déterminer la température seuil de croissance à partir de la teneur en azote réduit et de la VRC de plantes poussant à régime ther- 


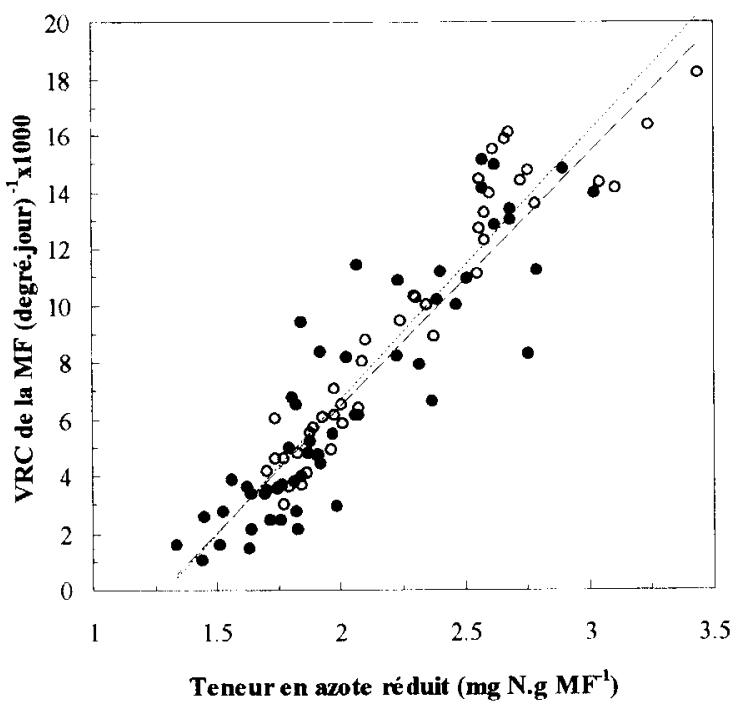

Fig 4. Relation entre la vitesse relative de croissance de la matière fraîche en (degré.jour) ${ }^{-1}$ (calculé avec une température seuil de $3{ }^{\circ} \mathrm{C}$ ) et la teneur en azote réduit dans la matière fraîche chez la laitue cultivée sous tunnel en été (symbole ouvert) ou en automne (symbole fermé), sur pot avec solution nutritive ; les pentes et les coefficients de détermination des droites de régression pour l'été (-) et l'automne (- - ) sont respectivement de 9,407 (es $=0,479$, dd $\left.=42, r^{2}=0,90\right)$ et de $8,908\left(\mathrm{es}=0,615, \mathrm{ddl}=50, r^{2}=0,80\right) ; p<0,001$.

mique différent, même si les plantes poussent sous un niveau de rayonnement différent. Ceci est lié à l'absence d'effet du niveau de rayonnement sur la relation entre vitesse relative de croissance et la teneur en azote réduit. Nos résultats fournissent une température seuil de $3^{\circ} \mathrm{C}$ ce qui permet de proposer une mesure du temps en degré. jour pour la laitue. L'utilisation des degrés.jour pour exprimer la vitesse relative de croissance en fonction de la teneur en azote réduit permet alors d'unifier des relations différentes obtenues dans des conditions climatiques variées. La prise en compte de la teneur en azote réduit (variable interne) et de la température (variable externe) explique alors près de $86 \%$ de la croissance en matière fraîche chez la laitue. La relation établie entre la vitesse relative de croissance exprimée en degré. jour et la teneur en azote réduit dans la matière fraîche peut alors servir de base pour l'élaboration d'un modèle reliant l'état azoté de la plante à sa croissance en matière frâiche.

\section{REMERCIEMENTS}

Nous remercions le CTIFL d'avoir accueilli la mise en place des expérimentations sur son centre de Lanxade, ainsi que J Marchal (Cirad-Flhor) pour avoir réalisé dans son laboratoire le dosage de l'azote total et du nitrate des échantillons de laitue. Ce travail a été finan- cé par le ministère de l'Agriculture (DGER) dans le cadre de l'action « Formation pour la recherche ».

\section{RÉFÉRENCES}

Bensink J (1971) On morphogenesis of lettuce leaves in relation to light and temperature. Meded Landbouwhogesch Wageningen nederland, 93 p

Caloin M, Yu O (1984) Analysis of time course of change in nitrogen content in Dactylis glomerata L. using a model of plant growth. Ann Bot 54, 69-76

Dapoigny L, Robin P, Raynal-Lacroix C, Fleury A (1996) Relation entre la vitesse relative de croissance et la teneur en azote chez la laitue (Lactuca sati$v a \mathrm{~L}$ ) : effets de l'ombrage et du niveau d'alimentation minérale. agronomie 16, 529-539

Fleury A, Bertrand M, Ronsin T (1986) Essai d'analyse climatique de la croissance précoce du maïs. $C R$ Acad Agric Fr 72, 591-602

Gosse G, Varlet-Grancher C, Bonhomme R, Chartier M, Allirand JM, Lemaire G (1986) Production maximale de matière sèche et rayonnement solaire intercepté par un couvert végétal. agronomie 6, 47-56

Greenwood DJ, Neeteson JJ, Draycott A (1986) Quantitative relationships for the dependence of growth rate of arable crops on the nitrogen content, dry weight and aerial environment. In : Fundamental, Ecological and Agricultural Aspects of Nitrogen Metabolism in Higher Plants ( $\mathrm{H}$ Lambers, JJ Neeteson, I Stulen, eds), Martinius Nijhoff, Boston, MA, 367-387

Greenwood DJ, Stone DA, Draycott A (1990) Weather, nitrogen-supply and growth rate of field vegetables. In : Plant Nutrition-Physiology and Applications (ML Van Beusichem, AD Van Diest, eds), Kluwer Academic Publishers, Dordrecht, 693-697

Greenwood DJ, Gastal F, Lemaire G, Draycott A, Millard P, Neeteson JJ (1991) Growth rate and \% N of field grown crops: theory and experiments. Ann Bot 67, 181-190

Ingestad T, McDonald AJS (1989) Interaction between nitrogen and photon flux density in birch seedlings at steady-state nutrition. Physio Plant 77, 1-11

Lawlor DW, Boyle FA, Keys AJ, Kendall AC, Young AT (1988) Nitrate nutrition and temperature effects on wheat: a synthesis of plant growth and nitrogen uptake in relation to metabolic and physiological processes. J Exp Bot 39, 329-343

Lemaire G, Salette J (1981) Analyse de l'influence de la température sur la croissance de printemps de graminées fourragères. C R Acad Sci Paris 292, 843846

Lemaire G, Salette J (1984) Relation entre dynamique de croissance et dynamique de prélèvement d'azote pour un peuplement de graminées fourragères. 1 . Étude de l'effet du milieu. agronomie 4, 423-430 
Lorenz HP, Wiebe HJ (1980) Effect of temperature on photosynthesis of lettuce adapted to different light and temperature conditions. Sci Horti 13, 115-123

Pontallier JY (1990) A cheap quantum sensor using a gallium arsenide photodiode. Functionnal Ecol 4 , $591-596$

Scaife MA (1973) The early relative growth rates of six lettuce cultivars as affected by temperature. Ann Appl Biol 74, $119-128$

Scaife A. Cox EF, Morris GEL (1987) The relation between shoot weight, plant density and time during the propagation of four vegetable species. Ann Bot 59 . 325-334

Venus JC, Causton DR (1979) Plant growth analysis: a reexamination of the methods of calculation of relat- tive growth and net assimilation rates without using fitted functions. Ann Bot 43, 633-638

Wheeler TR, Hadley P, Morison JIL, Ellis RH (1993a) Effects of temperature on the growth of lettuce (Lactuca sativa $\mathrm{L}$ ) and the implications for assessing the impacts of potentiel climate change. Eur J Agron $2,305-311$

Wheeler TR, Hadley P, Ellis RH, Morison JIL (1993b) Changes in growth and radiation use by lettuce crops in relation to temperature and ontogeny. Agri Forest Meteor 66, 173-186

Wurr DCE, Fellows JR (1984) The growth of three crisp lettuce varieties from different sowing dates. $J$ Agri Sci Camb 102, 733-745 